\title{
Theory of a Kaptiza-Dirac Interferometer with Cold Trapped Atoms
}

\author{
Ruolei Cheng1, Tianchen $\mathrm{He}^{2}$, Weidong Li ${ }^{1}$ Augusto Smerzi ${ }^{3}$ \\ ${ }^{1}$ Institute of Theoretical Physics and Department of Physics, Shanxi University, Taiyuan, China \\ ${ }^{2}$ Department of Physics, Taiyuan Normal University, Taiyuan, China \\ ${ }^{3} \mathrm{QSTAR}, \mathrm{INO}-\mathrm{CNR}$ and LENS, Firenze, Italy \\ Email: wdli@sxu.edu.cn
}

How to cite this paper: Cheng, R.L., He, T.C., Li, W.D. and Smerzi, A. (2016) Theory of a Kaptiza-Dirac Interferometer with Cold Trapped Atoms. Journal of Modern Physics, 7, 2043-2062.

http://dx.doi.org/10.4236/jmp.2016.715180

Received: October 13, 2016

Accepted: November 7, 2016

Published: November 10, 2016

Copyright $\odot 2016$ by authors and Scientific Research Publishing Inc. This work is licensed under the Creative Commons Attribution International License (CC BY 4.0).

http://creativecommons.org/licenses/by/4.0/ (c) (i) Open Access

\begin{abstract}
We theoretically analyse a multi-modes atomic interferometer consisting of a sequence of Kapitza-Dirac pulses (KD) applied to cold atoms trapped in a harmonic trap. The pulses spatially split the atomic wave-functions while the harmonic trap coherently recombines all modes by acting as a coherent spatial mirror. The phase shifts accumulated among different $\mathrm{KD}$ pulses are estimated by measuring the number of atoms in each output mode or by fitting the density profile. The sensitivity is rigorously calculated by the Fisher information and the Cramér-Rao lower bound. We predict, with typical experimental parameters, a temperature independent sensitivity which, in the case of the measurement of the gravitational constant $g$ can significantly exceed the sensitivity of current atomic interferometers.
\end{abstract}

\section{Keywords}

Atom Interferometry, Kapitza-Dirac Pulse, Dynamics, Gravitational Acceleration Constant, Sensitivity, Noise and Decoherence

\section{Introduction}

The goal of interferometry is to estimate the unknown value of a phase shift. The phase shift can arise because of a difference in length among two interferometric arms, as in the first optical Michelson-Morley probing the existence of aether or in LIGO and VIRGO gravitational wave detectors [1]. Phase shifts can also be the consequence of a supersonic airflow perturbing one optical path, as in the first Mach-Zehnder [2], or inertial forces as in Sagnac [3]. Interferometers are among the most exquisite measurement devices and since their first realisations have played a central role on pushing the frontier of science. 
Since the last decade, matter wave interferometers have progressively become very competitive when measuring electromagnetic or inertial forces. In particular, atom interferometers [4] [5] have been exploited to obtain the most accurate estimate of the gravitational constant [6] [7] [8] [9]. The beam splitter and the mirror operations of an atom interferometer can be typically implemented in free space with a sequence of Bragg scatterings applied to a beam of cold atoms [5] [10]. Alternatively, the phase shifts can be estimated by measuring the Bloch frequency of cold atoms oscillating in vertically oriented optical lattices which have been able to evaluate the gravitational constant $g$ with accuracy up to $\Delta g / g \sim 10^{-7}$ [11] [12] [13] [14].

The sensitivity of light-pulse atom interferometry scales linearly with the space-time area enclosed by the interfering atoms. Large-momentum-transfer (LMT) beam splitters have been suggested [15] and experimentally investigated [16] [17] [18], demonstrating up to $88 \hbar k$ splitting (where $\hbar k$ is the photon momentum) [16] [18]. Relative to the 2-photon processes used in the current most sensitive light-pulse atom interferometers, LMT beam splitters in atomic fountains can provide a 44 -fold increased phase shift sensitivity [16]. Further increases of the momentum differences between the interferometer paths are limited by the cloud's transverse momentum width since high efficiency beam splitting and mirror processes require a narrow distribution [19].

As an alternative to the atomic fountains, where the atoms follow ballistic trajectories, the interferometric operations can be implemented with trapped clouds [20] [21] [22]. We have recently proposed [23] a multi-mode interferometer with harmonically confined atoms where multi beam-splitter and mirror operations are realized with Kapitza-Dirac (KD) pulses, namely, the impulse application of an off-resonant standing optical wave. With KD pulses applied to atoms in a harmonic trap, it is possible to reach large spatial separations between the interferometric modes by avoiding, at the same time, atom losses and defocusing occurring in Bragg processes (mostly due to the constraint of narrow momentum widths). In [23], the role of mirrors is played by the harmonic trap, which coherently drives and recombines a tunable number of spatially addressable atomic beams created by the KD pulses. The phase estimation sensitivity linearly increases with the number of beams and their spatial distance. The number of beams is proportional to the strength of the applied KD pulse while their distance is proportional to the ratio between the harmonic trap length and the wave-length of the optical wave. In this manuscript we discuss in detail the theory of the multi-modes KD interferometer which was introduced in [23].

\section{Multi-Modes Kaptiza-Dirac Interferometer}

The initial configuration of the interferometer is provided by a cloud of cold atoms trapped by an harmonic potential $V_{h o}=m \omega^{2} x^{2} / 2$. The interferometric sequence is realised in four steps, see Figure 1:

i) Beam-splitter: A KD pulse is applied to the atomic cloud state at the time $t_{0} . \mathrm{KD}$ creates a number of spatially addressable atomic wave-packets that evolve along different paths under the harmonic confinement. 


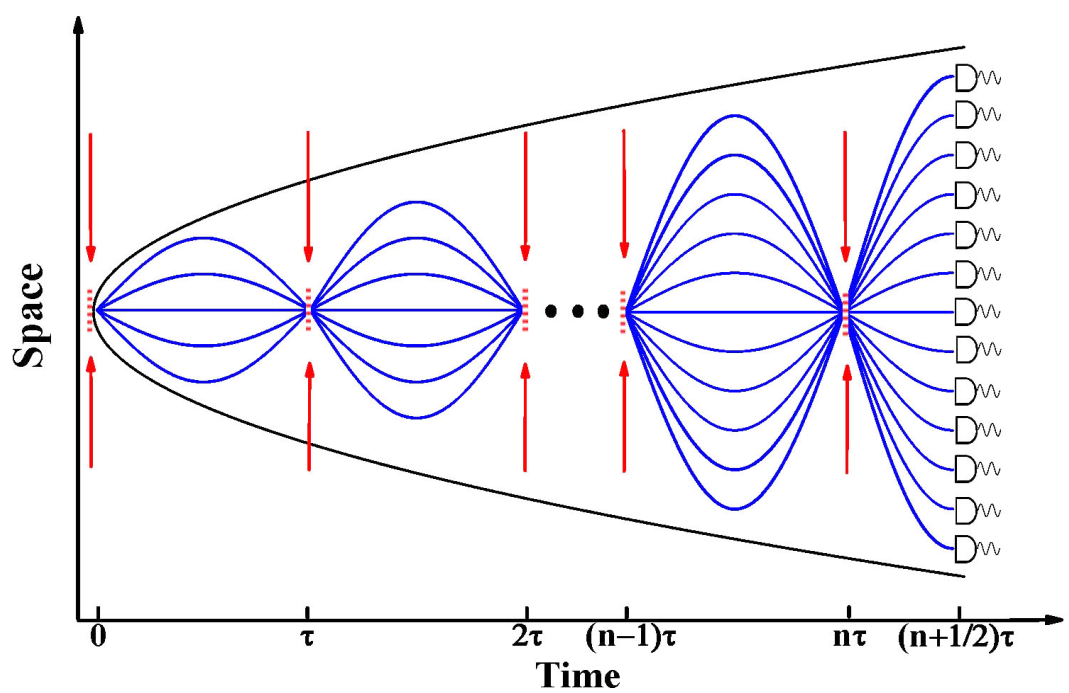

Figure 1. (color-online) Multimodes Kapitza-Dirac interferometer. The first Kapitza-Dirac pulse at $t=0$ creates several modes consisting of atomic wave-packets evolving under the harmonic confinement and an external perturbing field. The $n$-th Kapitza-Dirac pulse at $t=n \tau$ mixes the modes which are eventually detected in output at $t=(n+1 / 2) \tau$, where $\tau=\pi / \omega$.

ii) Phase shift: Each spatial mode gains a phase shift $\theta$ with respect to its neighbour's modes due to the action of an external potential.

iii) Beam splitter: the harmonic trap coherently recombines the wave packets and a second KD pulse is applied to again mix and separate the modes along different paths.

iv) Measurement: The phase shift is estimated by fitting the atomic density profile or by counting the number of atoms in each spatial mode at $t_{f}$. The measurement can be done after ballistic expansion by optimising spatial separation of the modes and atom counting signal to noise ratio.

The sequences i)-iii) can be iterated an arbitrary number of times $n$ before the final measurement iv).

The plan of the paper is as follows. In Section 2, we present a detailed description of the multi-modes KD interferometer. As an application we calculate the Fisher information and the Cramér-Rao lower bound sensitivity [24] of the interferometric measurement of the gravitational constant $g$ in Section 3. We predict sensitivities up to $\Delta g / g \sim 10^{-9}$ in configurations realisable within the current state of the art and in the Section 4 we compare the performance of different atomic interferometers. In Section 5 we discuss two possible sources of noise and we finally summarise the results in Section 6.

\section{Dynamics}

Let's consider first a single atom described by a wave packet $\psi_{0}(x)$ confided in the harmonic trap $V_{h}(x)=m \omega^{2} x^{2} / 2$. The time evolution of the state in the harmonic trap is given by

$$
\psi(x, t)=\int_{-\infty}^{+\infty} K\left(x, t ; y, t_{0}\right) \psi\left(y, t_{0}\right) \mathrm{d} y
$$


where $K\left(x, t ; y, t_{0}\right)$ is the quantum propagator [25]

$$
K\left(x, t ; y, t_{0}\right)=\frac{1}{\sqrt{i 2 \pi \sigma_{h}^{2} \sin \left(\omega\left(t-t_{0}\right)\right)}} \times \mathrm{e}^{\frac{i\left(\left(x^{2}+y^{2}\right) \cos \left(\omega\left(t-t_{0}\right)\right)-2 x y\right)}{2 \sigma_{h}^{2} \sin \left(\omega\left(t-t_{0}\right)\right)}},
$$

with $\sigma_{h}=\sqrt{\hbar / m \omega}$. The KD beam-splitter is realized with an impulse application of a periodic potential $V_{K D}(x)=V_{0} E_{r} \cos (k x)$, where $V_{0}$ is the strength of the pulse, $E_{r}$ is the atomic recoil energy and $k=4 \pi / \lambda$. In the Raman-Nath limit [10] [26] [27], the duration of the pulse is short enough to not affect the atomic density but to only change the phase of the initial wave-function $\psi(x, 0)$ as

$$
\psi(x)=\mathrm{e}^{-i V \cos (k x)} \psi_{0}(x)=\sum_{l=-\infty}^{+\infty} i^{l} J_{l}(-V) \mathrm{e}^{i l k x} \psi_{0}(x),
$$

where we have used the Bessel generating function $\mathrm{e}^{\frac{1}{2} z\left(t-\frac{1}{t}\right)}=\sum_{l=-\infty}^{+\infty} t^{l} J_{l}(z)$ [28] and $V=V_{0} E_{r} \delta t / \hbar$. The Raman-Nath limit has been experimentally demonstrated in [21] [29]. Equation (3) shows that the $\mathrm{KD}$ beam-splitter creates $M \simeq 2 V+1$ copies of the initial state, each with amplitude $J_{l}(-V)$ and an additional momentum $l k$.

After the application of the first $\mathrm{KD}$, the wave-packets are coherently driven by the harmonic trap and recombined after a time $\tau=\pi / \omega$. At this time, the propagator $K\left(x, t ; y, t_{0}\right)$ in Equation (2) is simply given by

$$
K_{1}\left(x, t_{0}+\tau ; y, t_{0}\right)=\mathrm{e}^{-i \frac{\omega \tau}{2}} \delta(x+y) .
$$

Furthermore, in presence of an external field, each spatial mode created by the KD beam splitter gains during the time $\tau$ a phase shift $\theta$ with respect to its neighbour's modes. Right before the application of a second KD pulse, at time $\tau_{-}$, the wave function is

$$
\psi\left(x, \theta, \tau_{-}\right)=\mathrm{e}^{-i \frac{\omega \tau}{2}} \mathrm{e}^{-i V \cos (k x-\theta)} \psi_{0}(-x) .
$$

After iterating a number of times $n$ the sequence of KD pulses and phase shift accumulations, the wave function at $t=n \tau_{-}$becomes

$$
\psi\left(x, \theta, n \tau_{-}\right)=\mathrm{e}^{-i \frac{n \omega \tau}{2}} \mathrm{e}^{-i V \Theta(n, \theta, x)} \psi_{0}\left((-)^{n} x\right),
$$

where

$$
\Theta(n, \theta, x)=\left[\frac{n}{2}\right] \cos (k x-\theta)+\left(n-\left[\frac{n}{2}\right]\right) \cos (k x+\theta),
$$

and $\left[\frac{n}{2}\right]$ is the integer part of $\frac{n}{2}$. For odd $n$ we have

$$
\Theta(n, \theta, x)=(n-1) \cos (\theta) \cos (k x)+\cos (k x-\theta),
$$

while for even $n$ we have

$$
\Theta(n, \theta, x)=n \cos (\theta) \cos (k x) .
$$


After $n$ iterations, a last $\mathrm{KD}$ pulse is applied at the time $n \tau$ providing

$$
\psi(x, \theta, n \tau)=\mathrm{e}^{-i V \cos (k x)} \psi\left(x, \theta, n \tau_{-}\right) .
$$

The wave packets gain their maximum spatial separation after a further $\tau / 2$ evolution in the harmonic trap.

Eventually, the wave function at the final time $t_{f}=(n+1 / 2) \tau$ right before measurement is

$$
\begin{aligned}
\psi\left(x, \theta, t_{f}\right) & =\int_{-\infty}^{+\infty} K_{2}\left(x, t_{f} ; y, n \tau\right) \psi(y, \theta, n \tau) \mathrm{d} y \\
& =\mathrm{e}^{i \phi\left(t_{f}\right)} \sum_{l=-\infty}^{+\infty} \mathrm{e}^{i \phi_{0}} i^{l} J_{l}[V(\theta)] \tilde{\psi}_{0}\left(\frac{x}{\sigma_{h}^{2}}-l k\right)
\end{aligned}
$$

where

$$
K_{2}\left(x, t_{0}+\frac{\tau}{2} ; y, t_{0}\right)=\frac{1}{\sqrt{2 \pi \sigma_{h}^{2}}} \mathrm{e}^{-i \frac{\omega \tau}{4}} \mathrm{e}^{-i \frac{x}{\sigma_{h}^{2}} y}
$$

and

$$
\tilde{\psi}_{0}\left(\frac{x}{\sigma_{h}^{2}}-l k\right)=\frac{1}{\sqrt{2 \pi \sigma_{h}^{2}}} \int_{-\infty}^{+\infty} \psi_{0}\left((-1)^{n} y\right) \mathrm{e}^{-i \frac{x-l k \sigma_{h}^{2}}{\sigma_{h}^{2}} y} \mathrm{~d} y
$$

with $\phi\left(t_{f}\right)=-(2 n+1) \omega \tau / 4, \quad \phi_{0}=l \beta$ or 0 for odd or even $n$, respectively, $\tan (\beta)=\frac{-\sin (\theta)}{n \cos (\theta)+1}$ and

$$
\begin{aligned}
& n \text { even }: V(\theta)=-V(n \cos (\theta)+1) \\
& n \text { odd }: V(\theta)=-V \sqrt{(n \cos (\theta)+1)^{2}+\sin ^{2}(\theta) .}
\end{aligned}
$$

In the limit if zero overlap between the various wave packets in Equation (8),

$$
\int_{-\infty}^{+\infty}\left|\tilde{\psi}_{0}\left(\frac{x-l k \sigma_{h}^{2}}{\sigma_{h}^{2}}\right)\right|^{2}\left|\tilde{\psi}_{0}\left(\frac{x-(l+1) k \sigma_{h}^{2}}{\sigma_{h}^{2}}\right)\right|^{2} \mathrm{~d} x=0,
$$

the density function at the measurement time $(2 n+1) \tau / 2$ simply becomes

$$
\rho\left(x, \theta, t_{f}\right)=\sum_{l=-\infty}^{+\infty} J_{l}^{2}[V(\theta)]\left|\tilde{\psi}_{0}\left(\frac{x}{\sigma_{h}^{2}}-l k\right)\right|^{2} .
$$

Equation (12) shows that there are $M=2 V(\theta)+1 \sim 2 n V+1$ momentum modes created by the $n$ applications of the KD pulses. This can of course be helpful if only weak KD pulses can be experimentally implemented.

In the limit of a large number $p \gg 1$ of independent interferometric measurements, the phase estimation sensitivity saturates the Cramér-Rao [30] lower bound

$$
\Delta^{2} \theta=\frac{1}{p N F},
$$


where $N$ is the number of uncorrelated atoms. $F$ denotes the Fisher information calculated from the particle density at the measurement time

$$
F=\int_{-\infty}^{+\infty} \frac{1}{\rho\left(x, \theta, t_{f}\right)}\left[\frac{\partial \rho\left(x, \theta, t_{f}\right)}{\partial \theta}\right]^{2} \mathrm{~d} x .
$$

With Equation (12), Equation (14) becomes

$$
F(\theta)=\left(\frac{\partial V(\theta)}{\partial \theta}\right)^{2} \sum_{l=-\infty}^{+\infty}\left[2 J_{l}^{\prime}(V(\theta))\right]^{2}=2\left(\frac{\partial V(\theta)}{\partial \theta}\right)^{2},
$$

(see Appendix). We finally obtain

$$
F(\theta)=2(n V)^{2} \sin ^{2}(\theta) S(n, \theta),
$$

where

$$
\begin{aligned}
& n \text { odd }: S(n, \theta)=1-\frac{1}{n^{2}}\left(\frac{n^{2}-2}{\sin ^{2}(\theta)+(n \cos (\theta)+1)^{2}}+1\right) \\
& n \text { even }: S(n, \theta)=1
\end{aligned}
$$

Notice that even in the case of a odd value of $n$, with $n \gg 1, S(n, \theta) \rightarrow 1$. Therefore, for an even $n$ or an odd $n \gg 1$, the phase estimation uncertainty of our interferometer becomes:

$$
\Delta \theta=\frac{1}{\sqrt{F(\theta)}} \frac{1}{\sqrt{p N}}=\frac{1}{|\sin (\theta)|} \frac{1}{\sqrt{2} n V} \frac{1}{\sqrt{p N}},
$$

which can also be written as

$$
\Delta \theta=\frac{\sqrt{2}}{|\sin (\theta)|} \frac{1}{M-1} \frac{1}{\sqrt{p N}},
$$

since the total number of modes is $M=2 n V+1$. As expected on a general ground from the theory of multimode interferometry [31], the sensitivity scales linearly with the number of momentum modes which have been significantly populated after KD beam splitters. The populations of higher diffraction orders vanish exponentially [10].

We remark here the important condition of non overlap of the wave packets corresponding to the different momentum modes at the time of measurement, Equation (11). A further interesting point is that Equation (18) is independent from the temperature of the atoms as long as their de Broglie wavelength remains larger than the internal spatial separation of the periodic potential creating the Kapitza-Dirac pulse. We will show this in the following Sections by considering as a specific application the interferometric estimation of the gravitational constant.

\section{Estimation of the Gravitational Acceleration Constant g}

We now investigate the KD interferometer theory to estimate the gravity constant $g$. The evolution of the initial state $\psi_{0}(x)$ is influenced by the combined action of the 
harmonic confinement $V_{h}(x)$, the gravitational field $m g x$ and the KD beam splitters. The goal is to estimate the value of the acceleration constant $g$. As explained in the previous Section, the phase shift $\theta$ arises from the external gravitational field acting during the phase accumulation period (until $n \tau$ ). We may engineer our Hamiltonian to switch on/off the gravity after the first beam splitter by modifying the frequency of the harmonic trap by $d=g\left(1 / \omega^{2}-1 / \tilde{\omega}^{2}\right)$, where $\omega, \tilde{\omega}$ are the trap frequencies before and after the $\mathrm{KD}$, respectively. We finally generalize our results by considering an atomic gas in thermal equilibrium at a finite temperature $T>0$.

To take in account the effect of the gravitational force on the dynamical evolution of the trapped atom states, we need to include in the free propagator $K\left(x, t ; y, t_{0}\right)$ Equation (2) the linear gravitational field [25]

$$
K_{g}\left(x, t ; y, t_{0}\right)=\mathrm{e}^{i \frac{\omega\left(t-t_{0}\right)}{2}\left(\frac{d}{\sigma_{h}}\right)^{2}} K\left(x^{\prime}, t ; y^{\prime}, t_{0}\right),
$$

where $x^{\prime}=x+d$ and $y^{\prime}=y+d$ with $d=g / \omega^{2}$. After the application of the first $\mathrm{KD}$, the states are coherently driven by the harmonic trap and the external gravitational field. At the time $t=\tau$, each spatial modes, created by KD pulse, are recombined and the wave function becomes

$$
\psi\left(x, g, \tau_{-}\right)=\mathrm{e}^{i \frac{\omega \tau}{2}\left[\left(\frac{d}{\sigma_{h}}\right)^{2}-1\right]} \mathrm{e}^{-i V \cos [k(x+2 d)]} \psi_{0}(-x-2 d),
$$

since the quantum propagator undergone with gravity field is reduced to

$$
K_{1, g}\left(x, t_{0}+\tau ; y, t_{0}\right)=\mathrm{e}^{i \frac{\omega \tau}{2}\left(\frac{d}{\sigma_{h}}\right)^{2}} K_{1}\left(x^{\prime}, t_{0}+\tau ; y^{\prime}, t_{0}\right) .
$$

As expected, each spatial mode gains its phase shift $2 d$ with respect to its neighbour's modes at time $\tau$ due to action of the external gravity field after the first KD pulse. A straightforward (slightly tedious) calculation provides the wave function at $t=n \tau_{-}$

$$
\psi\left(x, g, n \tau_{-}\right)=\mathrm{e}^{i \frac{n \omega \tau}{2}\left[\left(\frac{d}{\sigma_{h}}\right)^{2}-1\right]} \mathrm{e}^{-i V \Theta(n, g, x)} \psi_{0}(\zeta),
$$

where $\zeta=x$ for even $n$ and $\zeta=-x-2 d$ for odd $n$. The function $\Theta(n, g, x)$ also depends on the of $n$ : for odd $n$ we have

$$
\Theta(n, g, x)=(n-1) \cos (k d) \cos (k(x+d))+\cos (k(x+2 d)),
$$

and for even $n$

$$
\Theta(n, g, x)=n \cos (k d) \cos (k(x+d)) .
$$

The last KD pulse is applied on the wave function Equation (22) at time $n \tau$, to mix and therefore spatially separate the modes for the final density profile measurement

$$
\psi(x, g, n \tau)=\mathrm{e}^{-i V \cos (k x)} \psi\left(x, g, n \tau_{-}\right) .
$$

Firstly, we consider the case without the gravity field. Then at the time 
$t_{f}=n \tau+\tau / 2$, the wave function is

$$
\begin{aligned}
\psi\left(x, g, t_{f}\right) & =\int_{-\infty}^{+\infty} K_{2}\left(x, t_{f} ; y, n \tau\right) \psi(y, g, n \tau) \mathrm{d} y \\
& =\mathrm{e}^{i \Phi\left(t_{f}\right)} \sum_{l=-\infty}^{+\infty} i^{l} J_{l}[V(g)] \mathrm{e}^{-i \Phi_{0}} \tilde{\psi}_{0}\left(\frac{x}{\sigma_{h}^{2}}-l k\right),
\end{aligned}
$$

where $\Phi\left(t_{f}\right)=\frac{n \omega \tau}{2}\left(\frac{d}{\sigma_{h}}\right)^{2}+\phi\left(t_{f}\right)$ and for $n$ even (odd) $\Phi_{0}=l \beta \quad\left(\Phi_{0}=-l k d\right) . \beta$ is defined by

$$
\tan (\beta)=-\frac{n \sin (2 k d)}{n \cos (2 k d)+n+2},
$$

and $\tilde{\psi}_{0}\left(\frac{x-l k \sigma_{h}^{2}}{\sigma_{h}^{2}}\right)$ be found by replacing integral function $\psi_{0}\left((-1)^{n} y\right)$ as $\psi_{0}(\zeta)$ in Equation (10). Secondly, with gravity field, the wave function under quantum propagator with gravity field

$$
K_{2, g}\left(x, t_{0}+\frac{\tau}{2} ; y, t_{0}\right)=\mathrm{e}^{i \frac{d^{2} \omega \tau}{\sigma_{h}^{2}}} K_{2}\left(x^{\prime}, t_{0}+\frac{\tau}{2} ; y^{\prime}, t_{0}\right),
$$

where $K_{2}\left(x, t_{0}+\frac{\tau}{2} ; y, t_{0}\right)$ is defined by Equation (9), can be expressed as

$$
\begin{aligned}
\psi_{g}\left(x, g, t_{f}\right) & =\int_{-\infty}^{+\infty} K_{2, g}\left(x, t_{f} ; y, n \tau\right) \psi(y, g, n \tau) \mathrm{d} y \\
& =\mathrm{e}^{i\left(\frac{d^{2}}{\left.\sigma_{h}^{2} \frac{\omega \tau}{4}-\frac{(x+d) d}{\sigma_{h}^{2}}\right)} \psi\left(x^{\prime}, g, t_{f}\right),\right.}
\end{aligned}
$$

where $\psi\left(x, g, t_{f}\right)$ is defined by Equation (24) and

$$
\begin{aligned}
& n \text { even }: V(g)=-V \sqrt{\left[1+n(2+n) \cos ^{2}(k d)\right]}, \\
& n \text { odd }: V(g)=-(n+1) V \cos (k d) .
\end{aligned}
$$

Except the phase difference between Equation (24) and Equation (27), a constant difference $d$ is found in the centre position of each sub-wave packets induced by the gravity field. In the case of "no-overlap" condition (Equation (11)), which is satisfied when the width of the initial wave packet is much larger than the interwell distance of the KD optical lattice ( $\sigma_{0} / \lambda \gg 1$ ), the final density function becomes

$$
\rho\left(x, g, t_{f}\right)=\sum_{l=-\infty}^{+\infty} J_{l}^{2}[V(g)]\left|\tilde{\psi}_{0}\left(\frac{x}{\sigma_{h}^{2}}-l k\right)\right|^{2},
$$

from Equation (24) or

$$
\rho_{g}\left(x, g, t_{f}\right)=\sum_{l=-\infty}^{+\infty} J_{l}^{2}[V(g)] \mid \tilde{\psi}_{0}\left(\frac{x+d}{\sigma_{h}^{2}}-l k\right)^{2},
$$

from Equations ((27), (12) and (28)) show that the information on the estimated values 
of $\theta$ and $g$ are mainly (or entirely) contained in the weights $J_{l}^{2}[V(\theta, g)]$, depending on the final evolution during the measurement period. A small part of the information is involved in the center of sub-wave packets for half gravity evolution (Equation (29)).

We now consider an atomic gas at finite temperature $T$. To get some simple insight on the physics of the problem, we consider the system as made by a swarm of minimum uncertainty Gaussian wave packets

$$
\psi_{0}(x)=\frac{1}{\sqrt[4]{\pi \sigma_{0}}} \mathrm{e}^{-\frac{\left(x-x_{0}\right)^{2}}{2 \sigma_{0}^{2}}} \mathrm{e}^{i \frac{x p_{0}}{\hbar}}
$$

where the initial wave packet width $\sigma_{0}$ is equal to the thermal de Broglie wavelength $\lambda_{d B}=\sqrt{\frac{2 \pi \hbar^{2}}{m k_{B} T}}$ while the initial average coordinates $x_{0}$ and momentum $p_{0}$ are distributed according to the Boltzmann-Maxwell distribution

$$
f\left(x_{0}, p_{0}, T\right)=\frac{\omega}{2 \pi k_{B} T} \mathrm{e}^{-\frac{1}{k_{B} T}\left(\frac{p_{0}^{2}}{2 m}+\frac{1}{2} m \omega^{2} x_{0}^{2}\right)} .
$$

Each wav packet evolves driven by the propagators calculated in the previous Section:

$$
\tilde{\psi}_{0}\left(\frac{x}{\sigma_{h}^{2}}-l k\right)=\sqrt[4]{\frac{\sigma_{0}}{\pi}} \frac{1}{\sigma_{h}} \mathrm{e}^{i \varphi} \mathrm{e}^{\frac{-\sigma_{0}^{2}}{2}\left(\frac{x}{\sigma_{h}^{2}}-l k+(-1)^{n+1} \frac{p_{0}}{\hbar}\right)^{2}} \times \mathrm{e}^{i x_{0}\left((-1)^{n+1}\left(\frac{x}{\sigma_{h}^{2}}-l k\right)+\frac{p_{0}}{\hbar}\right)} .
$$

and

$$
\tilde{\psi}_{0}\left(\frac{x+d}{\sigma_{h}^{2}}-l k\right)=\sqrt[4]{\frac{\sigma_{0}}{\pi}} \frac{1}{\sigma_{h}} \mathrm{e}^{i \varphi} \mathrm{e}^{\frac{-\sigma_{0}^{2}}{2}\left(\frac{x+d}{\sigma_{h}^{2}}-l k+(-1)^{n+1} \frac{p_{0}}{\hbar}\right)^{2}} \times \mathrm{e}^{i x_{0}\left((-1)^{n+1}\left(\frac{x+d}{\sigma_{h}^{2}}-l k\right)+\frac{p_{0}}{\hbar}\right)} .
$$

where $\varphi=\frac{2 d\left(x-l k \sigma_{h}^{2}\right)}{\sigma_{h}^{2}}$ for odd $n$ and $\varphi=0$ for even $n$. Replacing in Equation (31), we find that the density distribution at the output of the interferometer is

$$
\begin{aligned}
\rho\left(x, g, t_{f}, T\right)= & \int_{-\infty-\infty}^{+\infty} \int_{-\infty}^{\infty} f\left(x_{0}, p_{0}, T\right)\left|\psi\left(x, g, t_{f}\right)\right|^{2} \mathrm{~d} x_{0} \mathrm{~d} p_{0} \\
= & \mathcal{N}(T) \sum_{l, l_{1}=-\infty}^{+\infty} i^{l}(-i)^{l_{1}} J_{l}[V(g)] J_{l_{1}}[V(g)] \\
& \times \mathrm{e}^{-\frac{1}{\xi^{2}(T)}\left[x-\frac{1}{2}\left(l+l_{1}\right) k \sigma_{h}^{2}\right]^{2}} \mathrm{e}^{-\Gamma(T)\left(I-l_{1}\right)^{2}}
\end{aligned}
$$

where $\mathcal{N}(T)=1 / \sqrt{\pi} \xi(T)$ is the normalization constant and

$$
\begin{gathered}
\xi^{2}(T)=\frac{4 \pi+1}{2 \pi} \frac{k_{B} T}{m \omega^{2}}, \\
\Gamma(T)=\frac{4 \pi^{2}}{\lambda^{2}}\left(\frac{2 k_{B} T}{m \omega^{2}}+\frac{2 \pi \hbar^{2}}{m k_{B} T}\right) .
\end{gathered}
$$

It is interesting to note that $\Gamma(T) \gg 16 \pi^{\frac{5}{2}}\left(\frac{\sigma_{h}}{\lambda}\right)^{2}$. In the case of $\sigma_{h} / \lambda \gg 1$, only the 
terms with $l=l_{1}$ in Equation (34) are important and the density profile at the final time reduces to a sum of weighted Gaussians of width $\xi(T)$ :

$$
\rho\left(x, g, t_{f}, T\right)=\mathcal{N}(T) \sum_{l=-\infty}^{+\infty} J_{l}^{2}\left[V_{(o, e)}(g)\right] \mathrm{e}^{-\frac{\left(x-l k \sigma_{h}^{2}\right)^{2}}{\xi^{2}(T)}},
$$

or

$$
\rho_{g}\left(x, g, t_{f}, T\right)=\rho\left(x^{\prime}, g, t_{f}, T\right) .
$$

Notice that the value of the gravitational constant $g$ is only contained in the weights of the modes.

The requirement is that sub-wave packets in Equation (37) are spatially separated, which means $\xi(T) \ll k \sigma_{h}^{2}$. Considering Equation (35), we have

$$
\frac{4 \pi+1}{2 \pi} \frac{k_{B} T}{m \omega^{2}} \ll k^{2} \sigma_{h}^{4},
$$

which means

$$
\left(\frac{\lambda_{d B}}{\lambda}\right)^{2} \gg \frac{4 \pi+1}{(4 \pi)^{2}}
$$

As expected, the spatial separation condition in Equation (37) $\xi(T) \ll k \sigma_{h}^{2}$ is equivalents to $\lambda_{d B} / \lambda \gg 1$. This means that the initial wave packets width (the thermal de Boglie wavelength) should be much larger than the internal distance of the KD potential. This is consistent with Equation (11). The important result is that as long as this condition is satisfied, the sensitivity does not depend on the temperature.

Substituting the density function Equation (37) at the measurement time $t_{f}$ into Fisher information Equation (14), we obtain

$$
F(T)=\int_{-\infty}^{+\infty} \frac{1}{\rho\left(x, g, t_{f}, T\right)}\left[\frac{\partial \rho\left(x, g, t_{f}, T\right)}{\partial g}\right]^{2} \mathrm{~d} x .
$$

The Fisher information for our system depends on the temperature, initial density profile, the interferometer transformation, and the choice of the observable that, here, is the spatial position of atoms. In this case, the estimator can simply be a fit of the final density profile. However, the same results would be obtained by choosing as observable, the number of particles in each Gassian spatial mode. Since the initial state is made of uncorrelated atoms, there is no need to measure correlations between the modes in order to saturate the Cramér-Rao lower bound Equation (13) at the optimal value of the value phase shift.

Before proceeding to discuss the finite temperature case, we calculate the highest sensitivity of the unbiased estimation of parameter $g$, which is guaranteed by the no-overlap condition $\lambda_{d B} / \lambda \gg 1$.

In the limit $T=0$, the Fisher information can be calculated analytically

$$
F=2\left(\frac{\partial V(g)}{\partial g}\right)^{2}=2 \frac{k^{2}}{\omega^{4}}(n V)^{2} \sin ^{2}(k d) S(n, g),
$$


where $S(n, g)=\frac{(n+2)^{2} \cos ^{2}(k d)}{1+n(n+2) \cos ^{2}(k d)}$ for even $n$ and $S(n, g)=(n+1)^{2} / n^{2}$ for odd $n$, with $S(n, g) \rightarrow 1$ in the limit $n \gg 1$. Finally, the Cramér-Rao lower bound Equation (13) becomes

$$
\frac{\Delta g}{g}=\frac{\omega^{2}}{g k} \frac{1}{\sqrt{2} n V} \frac{1}{|\sin (k d)|} \frac{1}{\sqrt{p N}},
$$

The Equation (43) can be rewritten as

$$
\frac{\Delta g}{g} \simeq \frac{\sqrt{2}}{2 \pi} \frac{\omega^{2} \lambda}{g} \frac{1}{M-1} \frac{1}{\sqrt{p N}} \simeq \frac{1}{\sqrt{2}} \frac{\hbar \omega}{m g L} \frac{1}{\sqrt{p N}}
$$

where $L=(M-1) k \sigma_{h}^{2}$.

If the gravity field is witched on in the last KD pulse, the density profile at final time is described by Equation (38). In this case, there is a further contribution to the Fisher Equation (42) from the shift on the center of sub-wave packets and we have

$$
F_{g}=\frac{2 m}{\hbar^{2} \omega^{3}}+F
$$

\section{Sensitivity}

We now estimate the expected sensitivity under realistic experiment conditions. We consider $10^{5}{ }^{88} \mathrm{Sr}$ atoms trapped in an harmonic trap having $\omega=2 \pi \times 5 \mathrm{~Hz}$ and a Kapitza-Dirac periodic potential with $\lambda=532 \times 10^{-9} \mathrm{~m}$ [21], recoil energy $E_{r}=h^{2} / 2 m \lambda^{2} \approx 5.33 \times 10^{-30} \mathrm{~J}$ and KD pulses applied for a time $\delta t=2 \times 10^{-6} \mathrm{~s}$.

With a strength of the KD potential $V=V_{0} E_{r} \delta t / \hbar \approx 4$ [21], a single pulse creates $\sim 9$ modes which provide a sensitivity with a single measurement shot and a phase accumulation time of 0.1 seconds, $\Delta g / g \sim 10^{-9}$. This sensitivity increases as $10^{-9} / \mathrm{n}$, see Equation (43), after $n$ pulses and phase accumulation time up to $n \times 0.1$ seconds. Under these conditions, the maximum length spanned by the ${ }^{88} \mathrm{Sr}$ atoms is also increased from $L_{1} \simeq 4.3 \times 10^{-3} \mathrm{~m}$ to $L_{n}=n \times L_{1}$, see the black lines in Figure 3 . In practice the sensitivity is limited by the effective length of the harmonic confinement. With current technologies using magnetic traps, the largest spatial separation $L$ could be pushed up to a few millimeters.

Since the thermal de Broglie wavelength decreases when increasing the temperature, the no-overlap condition Equation (11) breaks down at $k_{B} T_{0} \simeq 2 \pi\left(\sigma_{h} / \lambda\right)^{2}$. In Figure 2, we plot the normalised sensitivity as a function of the temperature. The time-independent sensitivity is found for various numbers of KD pulses. Once the temperature is increased up to the crossover value $T_{0}$, the sensitivity is drastically reduced see Figure 2. When $T<T_{0}$, the wave packets are spatially addressable (see dark and blue lines in Figure 3). When $T>T_{0}$, the distinguishability of the wave packets decreases (red lines in Figure 3) and the uncertainty in the phase estimation increases as $\Delta g \sim T$ for $T \gg T_{0}$. 


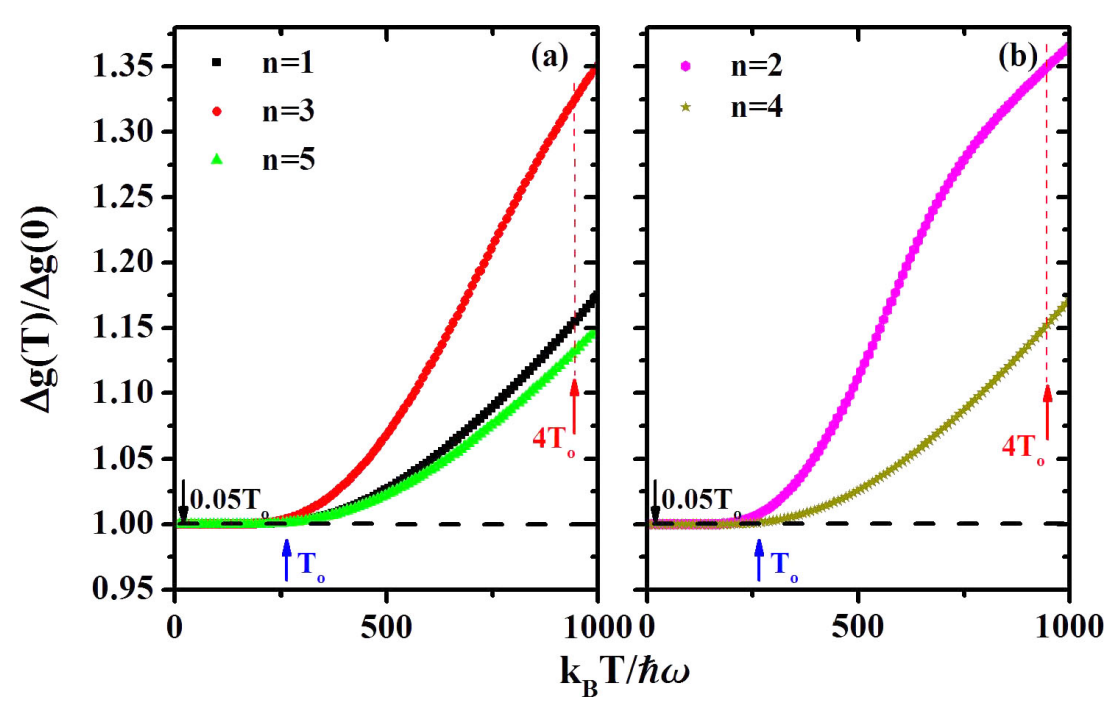

Figure 2. (color-online) Normalized phase estimation sensitivity as a function of the temperature for even and odd $n$.

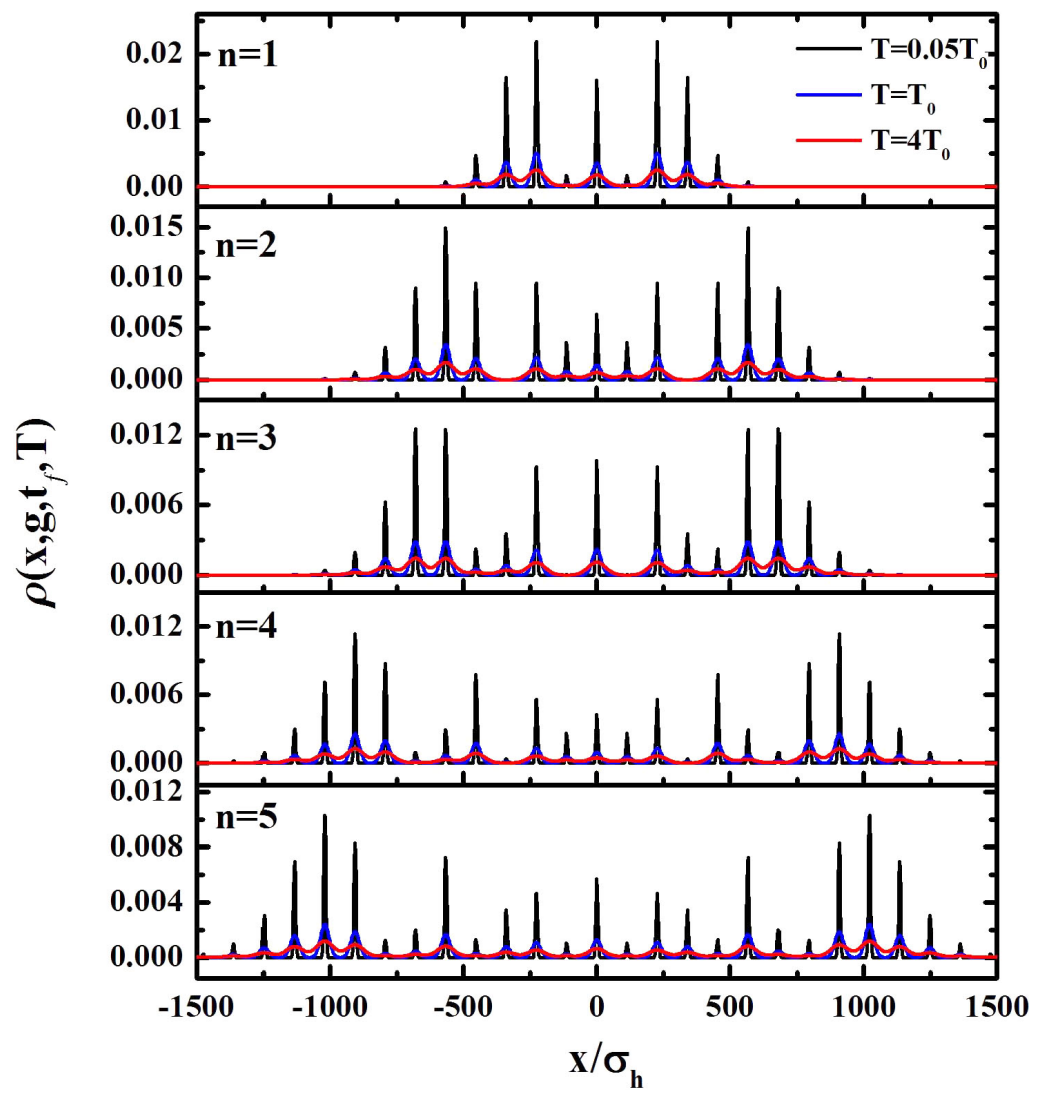

Figure 3. (color-online) Density profiles of the output wave function of Figure 2. The dark line, blue line and red line show temperatures below, equal and above the crossover temperature $T_{0}$.

As a comparison with current atom interferometers, we calculate the sensitivity obtained from a simple interference pattern observed after a free expansion of an initial atom clouds relevant, for instance, when measuring the gravitational constant $g$ using 
Bloch oscillations [12] [13] [14]. As shown in [32], the momentum distribution is expressed as

$$
\psi_{1}(p, \theta, t)=A \exp \left(-\frac{\sigma_{h}^{2} p^{2}}{2 \hbar^{2}}\right) \sum_{j=-\infty}^{+\infty} \exp \left(i j\left(\frac{p \lambda}{2 \hbar}+\theta\right)\right),
$$

where $\lambda$ is the wave length of the laser. $A$ is a normalization factor and $j$ denotes the lattice site and $\theta$ is the phase difference between lattice site. Since the finite size of the initial cold atomic cloud, there is only a finite number of terms in Equation (46) which contribute to the sum. We therefore have

$$
\sum_{j=-M^{\prime}}^{+M^{\prime}} \exp \left(i j\left(\frac{p \lambda}{2 \hbar}+\theta\right)\right)=\frac{\sin \left[\left(2 M^{\prime}+1\right)\left(\frac{p \lambda}{2 \hbar}+\theta\right)\right]}{\sin \left(\frac{p \lambda}{2 \hbar}+\theta\right)}
$$

where $2 M^{\prime}+1$ is the maximum numbers of the lattice occupied by the initial atom gases. In Equation (46), each point $\frac{2 \hbar}{\lambda}\left(\frac{2 \pi}{2 M^{\prime}+1}-\theta\right)$ has a Gaussian momentum distribution. Therefore, we obtain

$$
F=\int_{-\infty}^{+\infty} \frac{\left(\frac{\partial\left|\psi_{1}(p, \theta, t)\right|^{2}}{\partial \theta}\right)^{2}}{\left|\psi_{1}(p, \theta, t)\right|^{2}} \mathrm{~d} p=\frac{1}{6}\left(2 M^{\prime}\right)^{2} .
$$

where $A=\frac{\lambda}{4 \hbar \sqrt{3 \pi}}\left(2 M^{\prime}\right)$. Considering the experimental situations in [12] [13] [14], $\theta=m g \lambda t_{i n t} / 2 \hbar$, we arrive at

$$
F_{b l o}=\frac{m^{2} t_{i n t}^{2}\left[\left(2 M^{\prime}\right) \lambda / 2\right]^{2}}{6 \hbar^{2}}=\frac{m^{2} t_{\text {int }}^{2} \sigma_{h}^{2}}{6 \hbar^{2}} .
$$

where $t_{\text {int }}$ is the interaction time of the neighbour cold atom under the gravity-like force. Therefore it could be approximate as the tunnelling time $t_{\text {int }} \sim 1 / 3 \mathrm{~s}$ in [13]. With the Cramér-Rao lower bound Equation (13), we have

$$
\Delta^{2} g_{b l o}=\frac{6 \hbar^{2}}{m^{2} t_{\text {int }}^{2}\left(M^{\prime} \lambda\right)^{2}},
$$

where we have estimated the maximum occupied lattice sites by $\left(2 M^{\prime}+1\right) \lambda / 2 \sim \sigma_{h}$. Therefore, the sensitivity is

$$
\frac{\Delta g_{\text {blo }}}{g}=\frac{1}{g \sqrt{F_{\text {blo }}}} \frac{1}{\sqrt{p N}} \approx 10^{-6}
$$

Considering the sensitivity for a single Kaptiza-Dirac pulse with Equation (51), we can reach a sensitivity larger than 3 order of magnitude that the sensitivity obtained in an interference pattern. The reason is that the KD pulses can create several wave packets spanning a distance $\sim M k \sigma_{h}^{2}$, which can be quite a bit larger than the typical distances between the wave packets created in far field expansion measurements. In this 
case, the theoretical gain provided by Equation (43) is proportional to $M k \sigma_{h}^{2} / \sigma_{h}$, which can be $\sim 10^{3}$ with only once $\mathrm{KD}$ pulse and typical values of the experimental parameters. A further advantage is that such high sensitivity interferometry can be realised with a compact experimental setup.

\section{Noise and Decoherence}

We now consider the effects of noise and imperfections on the sensitivity of the interferometer. We mainly consider two kinds of perturbations, which may arise from the experimental realization of the interferometry. The first one is the effect of the anharmonicity, described by a position dependent random perturbation, and the second one the effect of a shift in position between different sequences of the KD pulses.

The effect of anharmonicity is investigated by numerically simulating the interferometric sequences with the following potential

$$
V(x)=V_{h}(x)+V_{R}(x),
$$

where $V_{R}(x)=V_{R} R(x) m \omega^{2} x^{2} / 2 . V_{R}$ is the strength of a position dependent random perturbation $R(x)$ having values $[-1,1]$. We take as length unit of the harmonic trap $\sigma_{h}$ and as time unit the inverse of the trap frequency $1 / \omega$. The strength of the external gravity-like potential is described by a dimensionless parameter $\alpha$, then $V_{e}=\alpha x$. To simplify the simulation, in the following we only consider a single KD pulse.

Starting with the ground state of the harmonic trap $V_{h}(x)$, the time dependent wave functions can be found by operator splitting method [33] with $\alpha=1$. Using $N_{1}$ groups of random numbers, we generate $N_{1}$ densities at the measurement time. Then, the average density $\rho_{\text {ave }}\left(x, \alpha, t_{f}\right)=\left[\sum_{j=1}^{N_{1}} \rho_{j}\left(x, \alpha, t_{f}\right)\right] / N_{1}$ is used for calculating Fisher information $F(R)_{\text {ave }}$ for given $N_{1}$. Here, we use the Equation (53) to get the derivative for $\alpha=1$ and $h=0.01$

$$
\frac{\mathrm{d} f(x)}{\mathrm{d} x}=\frac{1}{2 h}[-f(x-h)+f(x+h)]+O\left[h^{2}\right]
$$

Due to the perturbation potential $V_{R}(x)$, the sub-wave packets are driven back to their initial position with a incoherent phase at $t=\tau$ and the total density profile could be dramatically destructed. It is interesting to note that the KD pulses still do a quite good job and that completed spatially separated wave packets with momentum $l k$ can be found at the measurement time $t_{f}$, see Figure 4 . When increasing $N_{1}$, the visibility of the wave packets decreases compared with the ideal case (Black line, without $V_{R}(x)$ ). This definitely makes a impact on the sensitivity, which can be found by calculating the Fisher information $F(R)_{\text {ave }}$ through $\rho_{\text {ave }}\left(x, \alpha, t_{f}\right)$. The results have been presented in Figure 5. Generally speaking, a strong perturbation of the harmonic potential decreases dramatically, see Figure $5(\mathrm{~b})$, while, for $V_{R}<0.1 \mathrm{~V}$ it is still possible to obtain a sensitivity comparable with the ideal case.

A shift of the optical lattice with respect to the harmonic trap $V_{h}(x)$ is further possible reason for a decreased sensitivity. Assuming a off center shift of two consecu- 


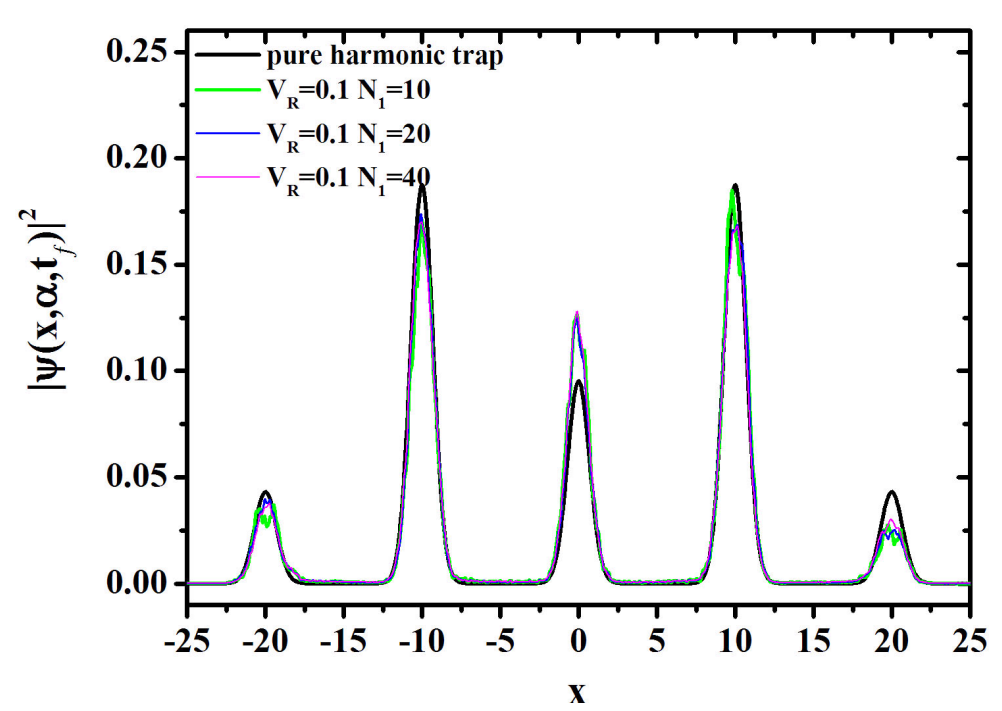

Figure 4. (color-online) Density profiles around each momentum component $l k \quad\left(V_{R}<0.1 \mathrm{~V}\right)$ at time $t_{f}=\frac{3 \pi}{2}$. The parameters are $V=1, \alpha=1, k=10$. The black line is for the pure harmonic trap. The green line is the average density $\rho_{\text {ave }}\left(x, \alpha, t_{f}\right)$ of ten groups of random number $\left(N_{1}=10\right)$. The blue line is for $N_{1}=20$. The pink line is for $N_{1}=40$.
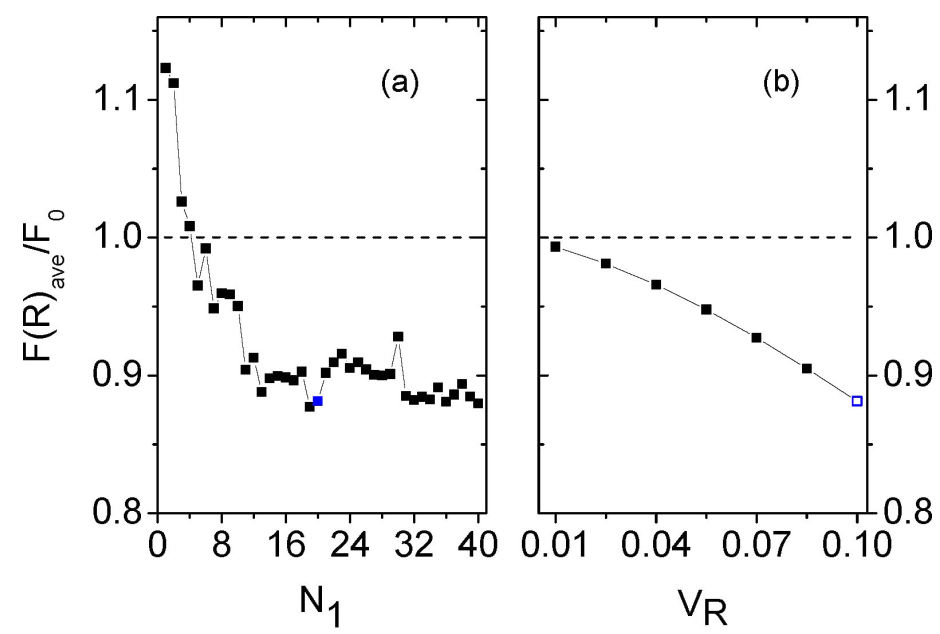

Figure 5. (color-online) The average Fisher information $F(R)_{a v e} / F_{0}$, with $V=1, \alpha=1, k=10$. (a) $V_{R}=0.1$, (b) $N_{1}=20$.

tive KD pulses $\delta x_{1,2}$, the wave function at $t=\tau$ after the second KD is

$$
\psi(x, g, \tau)=\mathrm{e}^{i \frac{\pi}{2}\left[\left(\frac{d}{\sigma_{h}}\right)^{2}-1\right]} \mathrm{e}^{i V(g) \cos \left[k\left(x+d-\delta x_{1} / 2+\delta x_{2} / 2\right)\right]} \times \psi_{0}(-x-2 d),
$$

where $V(g)=-2 V \cos \left[k\left(d-\left(\delta x_{1}+\delta x_{2}\right) / 2\right)\right]$. To get this result we have considered the properties of Bessel generating function [28].

$$
\psi\left(x, g, t_{f}\right)=\mathrm{e}^{i \phi\left(t_{f}\right)} \sum_{l=-\infty}^{+\infty} i^{l} J_{l}[V(g)] \mathrm{e}^{i l k\left(d-\delta x_{1} / 2+\delta x_{2} / 2\right)} \times \tilde{\psi}_{0}\left(\frac{x-l k \sigma_{h}^{2}}{\sigma_{h}^{2}}\right),
$$


where $\phi\left(t_{f}\right)=\frac{\pi}{2}\left[\left(\frac{d}{\sigma_{h}}\right)^{2}-1\right]-\frac{\pi}{4}$. Equation (55) shows that the effect of off-center S

i

$\mathrm{f}$

$\mathrm{t}$

makes only an phase shifts for each sub-wave packets. Therefore, the non-overlap condition Equation (11) does not have any modification even after considering the off-center shift In this case, the final density profiles is

$$
\left|\psi\left(x, g, t_{f}\right)\right|^{2}=\sum_{l=-\infty}^{+\infty} J_{l}^{2}[V(g)]\left|\tilde{\psi}_{0}\left(\frac{x-l k \sigma_{h}^{2}}{\sigma_{h}^{2}}\right)\right|^{2} .
$$

Equation (56) shows that the center shifts could induce a fluctuation by $\delta x_{1}+\delta x_{2}$ around the estimated value of $d$. If those off-center shifts are coming from some external noise, then it may do not play crucial effect on the value of $V(g)$. Therefore, it does have small effect on Fisher information, by

$$
F_{c}=2 \frac{k^{2}}{\omega^{4}}(2 V)^{2} \sin ^{2}\left[k d-k \frac{\delta x_{1}+\delta x_{2}}{2}\right] \text {. }
$$

\section{Conclusion}

During the last few decades, matter-wave interferometry has been successfully extended to the domain of atoms and molecules. Most current interferometric protocols for the measurement of gravity or inertial forces are based on the manipulation of free falling atoms realizing Mach-Zehnder like configurations. Here we propose an atomic multimode interferometer with atoms trapped in a harmonic potential and where the multi beam-splitter operation are implemented with Kapitza-Dirac pulses. The mirror operations are performed by the harmonic trap which coherently drives a tunable number of spatially addressable atomic beams. All interferometer processes, including splitting, phase accumulation and reflection are performed and completed within the harmonic trap. Therefore, all trapped atoms contribute to the sensitivity. We have applied our scheme to the estimation of the gravitational constant and estimate, with realistic experimental parameters, a sensitivity of $10^{-9}$, significantly exceeding the sensitivity of current interferometric protocols.

\section{Acknowledgements}

Our work is supported by the National Science Foundation of China (No. 11374197), PCSIRT (No. IRT13076), the National Science Foundation of China (No.11504215).

\section{References}

[1] Abbott, B.P., et al. (2016) Physical Review Letters, 116, Article ID: 241103. http://dx.doi.org/10.1103/PhysRevLett.116.241103

[2] Zehnder, L. (1891) Zt. Instrumentenkd, 11, 275.

[3] Culshaw, B. (2006) Measurement Science and Technology, 17, R1. http://dx.doi.org/10.1088/0957-0233/17/1/R01 
[4] Cronin, A.D., Schmiedmayer, J. and Pritchard, D.E. (2009) Reviews of Modern Physics, 81, 1051. http://dx.doi.org/10.1103/RevModPhys.81.1051

[5] Fixler, J.B., Foster, G.T., McGuirk, J.M. and Kasevich, M.A. (2007) Science, 315, 74-77. http://dx.doi.org/10.1126/science.1135459

[6] Rosi, G., Sorrentino, F., Cacciapuoti, L., Prevedelli, M. and Tino, G.M. (2014) Nature (London), 510, 518-521. http://dx.doi.org/10.1038/nature13433

[7] Biedermann, G.W., Wu, X., Deslauriers, L., Roy, S., Mahadeswaraswamy, C. and Kasevich, M.A. (2015) Physical Review A, 91, Article ID: 033629. http://dx.doi.org/10.1103/PhysRevA.91.033629

[8] Zhou, L., Long, S.T., Tang, B., Chen, X., Gao, F., Peng, W.C., Duan, W.T., Zhong, J.Q., Xiong, Z.Y., Wang, J., Zhang, Y.Z. and Zhan, M.S. (2015) Physical Review Letters, 115, Article ID: 013004. http://dx.doi.org/10.1103/PhysRevLett.115.013004

[9] Chaibi, W., Geiger, R., Canuel, B., Bertoldi, A., Landragin, A. and Bouyer, P. (2016) Physical Review D, 93, Article ID: 021101(R). http://dx.doi.org/10.1103/PhysRevD.93.021101

[10] Keller, C., Schmiedmayer, J., Zeilinger, A., Nonn, T., Dürr, S. and Rempe, G. (1999) Applied Physics B, 69, 303-309. http://dx.doi.org/10.1007/s003400050810

[11] Roati, G., de Mirandes, E., Ferlaino, F., Ott, H., Modugno, G. and Inguscio, M. (2004) Physical Review Letters, 92, Article ID: 230402. http://dx.doi.org/10.1103/PhysRevLett.92.230402

[12] Ferrari, G., Poli, N., Sorrentino, F. and Tino, G.M. (2006) Physical Review Letters, 97, Article ID: 060402. http://dx.doi.org/10.1103/PhysRevLett.97.060402

[13] Poli, N., Wang, F.-Y., Tarallo, M.G., Alberti, A., Prevedelli, M. and Tino, G.M. (2011) Physical Review Letters, 106, Article ID: 038501. http://dx.doi.org/10.1103/PhysRevLett.106.038501

[14] Tarallo, M.G., Alberti, A., Poli, N., Chiofalo, M.L., Wang, F.-Y. and Tino, G.M. (2012) Physical Review A, 86, Article ID: 033615. http://dx.doi.org/10.1103/PhysRevA.86.033615

[15] Denschlag, J.H., et al. (2002) Journal of Physics B: Atomic, Molecular and Optical Physics, 35, 3095. http://dx.doi.org/10.1088/0953-4075/35/14/307

[16] Müller, H., Chiow, S.-W., Long, Q., Herrmann, S. and Chu, S. (2008) Physical Review Letters, 100, Article ID: 180405. http://dx.doi.org/10.1103/PhysRevLett.100.180405

[17] Cladé, P., Guellati-Khélifa, S., Nez, F. and Biraben, F. (2009) Physical Review Letters, 102, Article ID: 240402. http://dx.doi.org/10.1103/PhysRevLett.102.240402

[18] Müller, H., Chiow, S.-W., Herrmann, S. and Chu, S. (2009) Physical Review Letters, 102, Article ID: 240403. http://dx.doi.org/10.1103/PhysRevLett.102.240403

[19] Szigeti, S.S., Debs, J.E., Hope, J.J., Robins, N.P. and Close, J.D. (2012) New Journal of Physics, 14, Article ID: 023009. http://dx.doi.org/10.1088/1367-2630/14/2/023009

[20] Garcia, O., Deissler, B., Hughes, K.J., Reeves, J.M. and Sackett, C.A. (2006) Physical Review $A$, 74, Article ID: 031601(R). http://dx.doi.org/10.1103/PhysRevA.74.031601

[21] Sapiro, R.E., Zhang, R. and Raithel, G. (2009) Physical Review A, 79, Article ID: 043630. http://dx.doi.org/10.1103/PhysRevA.79.043630

[22] Close, J. and Robins, N. (2012) Physics, 5, 26. http://dx.doi.org/10.1103/Physics.5.26

[23] Li, W.D., He, T.C. and Smerzi, A. (2014) Physical Review Letters, 113, Article ID: 023003. http://dx.doi.org/10.1103/PhysRevLett.113.023003

[24] Pezzé, L. and Smerzi, A. (2014) Atom Interferometry. Proceedings of the International School of Physics “Enrico Fermi”, Course 188, Societa' Italiana di Fisica, Bologna and IOS 
Press, Amsterdam.

[25] Holstein, B.R. (1992) Topics in Advanced Quantum Mechanics. Addison-Wesley, Reading, MA.

[26] Edwards, M., Benton, B., Heward, J. and Clark, C.W. (2010) Physical Review A, 82, Article ID: 063613. http://dx.doi.org/10.1103/PhysRevA.82.063613

[27] Moharam, M.G. and Young, L. (1978) Applied Optics, 17, 1757-1759. http://dx.doi.org/10.1364/AO.17.001757

[28] Abramowitz, M. and Stegun, I.A. (1970) Handbook of Mathematical Functions: With Formulas, Graphs and Mathematical Tables. National Bureau of Standards, Washington DC.

[29] Deng, L., Hagley, E., Denschlag, J., Simsarian, J., Edwards, M., Clark, C., Helmerson, K., Rolston, S. and Phillips, W. (1999) Physical Review Letters, 83, 5407. http://dx.doi.org/10.1103/PhysRevLett.83.5407

[30] Hyllus, P., Laskowski, W., Krischek, R., Schwemmer, C., Wieczorek, W., Weinfurter, H., Pezze, L. and Smerzi, A. (2012) Physical Review A, 85, Article ID: 022321. http://dx.doi.org/10.1103/PhysRevA.85.022321

[31] Chwedenczuk, J., Piazza, F. and Smerzi, A. (2013) Physical Review A, 87, Article ID: 033607. http://dx.doi.org/10.1103/PhysRevA.87.033607

[32] Pedri, P., Pitaevskii, L., Stringari, S., et al. (2001) Physical Review Letters, 87, Article ID: 220401. http://dx.doi.org/10.1103/PhysRevLett.87.220401

[33] Bandrauk, A.D. and Shen, H. (1994) Journal of Physics A: Mathematical and General, 27, 7147. http://dx.doi.org/10.1088/0305-4470/27/21/030 


\section{Appendix}

\section{Appendix A}

To obtain Equation (15), we have considered the Bessel functions identity

$$
J_{l}^{\prime}(V)=J_{l-1}(V)-J_{l+1}(V) .
$$

With this we have

$$
\begin{aligned}
\sum_{l=-\infty}^{+\infty}\left[2 J_{l}^{\prime}(V(\theta))\right]^{2}= & \sum_{l=-\infty}^{+\infty} J_{l-1}^{2}(V(\theta))+\sum_{l=-\infty}^{+\infty} J_{l+1}^{2}(V(\theta)) \\
& -2 \sum_{l=-\infty}^{+\infty} J_{l-1}(V(\theta)) J_{l+1}(V(\theta)) \\
= & 2,
\end{aligned}
$$

where one more identity

$$
\sum_{j=-\infty}^{+\infty} J_{j}(A) J_{v \mp j}(B)=J_{v}(B \pm A) .
$$

has been used to obtain

$$
\sum_{l=-\infty}^{+\infty} J_{l-1}(V(\theta)) J_{l+1}(V(\theta))=0
$$

\section{Appendix B}

For Equation (45), Using the Equation (41) and Equation (29), we obtain

$$
\begin{aligned}
\frac{\partial \rho_{g}\left(x, g, t_{f}\right)}{\partial g} & =\sum_{l=-\infty}^{+\infty} \frac{\partial J_{l}^{2}[V(g)]}{\partial g}\left|\tilde{\psi}_{0}\left(\frac{x+d}{\sigma_{h}^{2}}-l k\right)\right|^{2} \\
& +J_{l}^{2}[V(g)] \frac{\partial\left|\tilde{\psi}_{0}\left(\frac{x+d}{\sigma_{h}^{2}}-l k\right)\right|^{2}}{\partial g} .
\end{aligned}
$$

By using the initial state

$$
\psi_{0}(y)=\sqrt[4]{\frac{1}{\pi \sigma_{h}^{2}}} \mathrm{e}^{-\frac{y^{2}}{2 \sigma_{h}^{2}}}
$$

we get

$$
\begin{aligned}
& \tilde{\psi}_{0}\left(\frac{x+d-l k \sigma_{h}^{2}}{\sigma_{h}^{2}}\right)=\frac{1}{\sqrt{2 \pi \sigma_{h}^{2}}} \sqrt[4]{\frac{1}{\pi \sigma_{h}^{2}}} \int_{-\infty}^{+\infty} \psi_{0}(\zeta) \times \mathrm{e}^{-i \frac{x+d-l k \sigma_{h}^{2}}{\sigma_{h}^{2}} y} \mathrm{~d} y \\
& =\sqrt[4]{\frac{1}{\pi \sigma_{h}^{2}}} \mathrm{e}^{i \mu_{n}} \mathrm{e}^{-\frac{\left(x+d-l k \sigma_{h}^{2}\right)^{2}}{2 \sigma_{h}^{2}}} .
\end{aligned}
$$

where $\mu_{n}=0$ for even $n$, but $\mu_{n}=\frac{x+d-l k \sigma_{h}^{2}}{\sigma_{h}^{2}} 2 d$ for odd $n$. So 


$$
\begin{aligned}
F_{g} & =\int_{-\infty}^{+\infty} \frac{1}{\rho_{g}\left(x, g, t_{f}\right)}\left[\frac{\partial \rho_{g}\left(x, g, t_{f}\right)}{\partial g}\right]^{2} \mathrm{~d} x \\
& =\int_{-\infty}^{+\infty} \frac{1}{\rho_{g}\left(x, g, t_{f}\right)} \sum_{l=-\infty}^{+\infty}\left[J_{l}^{2}(V(g)) \times \frac{\partial\left|\tilde{\psi}_{0}\left(\frac{x+d}{\sigma_{h}^{2}}-l k\right)\right|^{2}}{\partial g}\right]^{2} \mathrm{~d} x+\sum_{l=-\infty}^{+\infty} \frac{\partial V(g)}{\partial g}\left[2 J_{l}^{\prime}\left(V_{g}\right)\right]^{2} \\
& =\frac{4}{\sigma_{h}^{4} \omega^{4}} \sum_{l=-\infty}^{+\infty} J_{l}^{2}(V(g)) \times \sqrt{\frac{1}{\pi \sigma_{h}^{2}} \int_{-\infty}^{+\infty} \mathrm{e}} \\
& =2 \frac{m}{\hbar^{2} \omega^{3}}+F .
\end{aligned}
$$

The second step uses the "no-overlap" condition by changing $x$ to $x^{\prime}$ in Equation (11).

Submit or recommend next manuscript to SCIRP and we will provide best service for you:

Accepting pre-submission inquiries through Email, Facebook, LinkedIn, Twitter, etc.

A wide selection of journals (inclusive of 9 subjects, more than 200 journals)

Providing 24-hour high-quality service

User-friendly online submission system

Fair and swift peer-review system

Efficient typesetting and proofreading procedure

Display of the result of downloads and visits, as well as the number of cited articles

Maximum dissemination of your research work

Submit your manuscript at: http://papersubmission.scirp.org/

Or contact jmp@scirp.org 